\title{
Regulation of intestinal myofibroblasts by KRas-mutated colorectal cancer cells through heparin-binding epidermal growth factor-like growth factor
}

\author{
HIDEYOSHI KAWASAKI ${ }^{1}$, TAKUYA SAOTOME ${ }^{1}$, TATSUYA USUI ${ }^{2}$, TAKASHI OHAMA ${ }^{1}$ and KOICHI SATO ${ }^{1}$ \\ Laboratories of ${ }^{1}$ Veterinary Pharmacology, ${ }^{2}$ Veterinary Toxicology, Joint Faculty of Veterinary Medicine, \\ Yamaguchi University, Yamaguchi 753-8515, Japan
}

Received December 22, 2016; Accepted February 28, 2017

DOI: $10.3892 /$ or.2017.5520

\begin{abstract}
In colorectal cancer, gain-of-function mutations in KRas play a critical role in malignant transformation. Tumor growth in colorectal cancer is known to be promoted by the intestinal myofibroblasts (IMFs) that localize adjacent to the cancer cells, but the mechanisms of interaction between KRasmutated cancer cells and the myofibroblasts remain unclear. Here, we investigated the effects of KRas-mutated cells on the behavior of myofibroblasts by using mouse primary IMFs and cells of an IMF cell line (LmcMF) and a mouse colon epithelial cell line $(\mathrm{aMoC} 1)$. Conditioned medium (CM) was collected from aMoC1 cells overexpressing a control vector or KRasV12 vector (KRasV12-CM), and the effects of KRasV12-CM on IMFs were analyzed by performing proliferation assays, wound-healing assays, Boyden chamber assays, and western blotting. Whereas KRasV12-CM exerted little effect on the differentiation and proliferation of primary IMFs, the CM promoted migration of both primary IMFs and LmcMF cells. In KRasV12-overexpressing aMoC1 cells, mRNA expression of heparin-binding epidermal growth factor-like growth factor (HB-EGF) was higher than in mock-transfected aMoC1 cells, and HB-EGF promoted the migration of primary IMFs and LmcMF cells. Moreover, KRasV12-CM-induced IMF migration was suppressed by dacomitinib, an inhibitor of HB-EGF receptors. Notably, in LmcMF cells, both KRasV12-CM and HB-EGF activated extracellular signal-regulated kinase (ERK) and c-jun N-terminal kinase (JNK), whereas KRasV12CM-induced migration of IMFs was suppressed following treatment with either an ERK inhibitor (FR180204) or a JNK inhibitor (SP600125). These results suggest that HB-EGF
\end{abstract}

Correspondence to: Professor Koichi Sato, Laboratory of Veterinary Pharmacology, Joint Faculty of Veterinary Medicine, Yamaguchi University, 1677-1 Yoshida, Yamaguchi 753-8515, Japan E-mail: k-sato@yamaguchi-u.ac.jp

Key words: cancer microenvironment, cell migration, colorectal cancer, heparin-binding epidermal growth factor-like growth factor, KRas, myofibroblast secreted from KRas-mutated colorectal cancer cells promotes IMF migration through ERK and JNK activation, which, in turn, could support cancer progression.

\section{Introduction}

Colorectal cancer is the third most common cancer in males and the second most common in females worldwide (1). In colorectal cancer, gain-of-function mutations in KRas are detected in approximately $40 \%$ of the tumors and play a critical role in malignant transformation (2). KRas mutations activate intracellular signals and stimulate tumor cell growth, invasion, metastasis, and drug resistance (3-6). Moreover, KRas mutations promote cytokine secretion in tumor cells, which might contribute to cancer initiation and progression (7-9).

In cancer tissues, several types of cells, such as myofibroblasts, immune cells, and vascular cells, form the tumor microenvironment and thus promote tumor progression (10). The tumor microenvironment contributes to cancer progression by promoting tumor cell growth, inhibiting apoptosis, enhancing angiogenesis, and suppressing antitumor immunity $(11,12)$. The tumor cells, in turn, regulate stromal cells through paracrine signaling and thereby generate an environment conducive to tumor progression $(13,14)$.

Intestinal myofibroblasts (IMFs) localized subjacent to the intestinal epithelium interact with epithelial cells and play crucial roles in maintaining epithelial homeostasis $(15,16)$. Myofibroblasts regulate various diseases, such as inflammation, fibrosis, and carcinogenesis. In cancer tissues, myofibroblasts localize adjacent to cancer stem cells and participate in mutual feedback signaling loops $(14,17)$. Myofibroblasts secrete cytokines such as interleukin-6 (IL-6), hepatocyte growth factor (HGF), and heparin-binding epidermal growth factor-like growth factor (HB-EGF) and thus promote tumor progression (17-20). Conversely, tumor cells secrete transforming growth factor- $\beta$ (TGF- $\beta$ ) and platelet-derived growth factor (PDGF), which regulate the migration, differentiation, and cytokine secretion of myofibroblasts $(18,21)$. Although these interactions have gradually come to be regarded as new therapeutic targets (22), the mechanisms of interaction between KRas-mutated cancer cells and myofibroblasts have remained unclear. 
In this study, to elucidate how KRas-mutated cancer cells interact with myofibroblasts, we investigated the molecular mechanisms of the regulation of IMFs by KRas-mutated cells.

\section{Materials and methods}

Mice. Male C57BL/6J mice (4-12 weeks old) were purchased from Charles River Japan (Yokohama, Japan) and maintained in accordance with the guidelines of the Animal Care and Use Committee of Yamaguchi University. Experimental protocols were approved by the Yamaguchi University Animal Care and Use Committee.

Cell culture and conditioned medium (CM) collection. Mouse IMFs were isolated as previously described (23). LmcMF, a mouse colon myofibroblast cell line, was established in our previous study (23). An adult mouse colon epithelial cell line, aMoC1, was provided by Dr Mamoru Totsuka (Tokyo University) (24). IMFs and LmcMF cells were cultured in Dulbecco's modified Eagle's medium (DMEM; Thermo Fisher Scientific, Waltham, MA, USA) containing $10 \%$ fetal bovine serum (FBS) and 1\% antibiotic-antimycotic solution (AA; Thermo Fisher Scientific), whereas aMoC1 cells were cultured in DMEM containing 5\% FBS, $1 \%$ insulin-transferrinselenium-X (Thermo Fisher Scientific), and 1\% AA solution. $\mathrm{CM}$ was obtained by collecting the supernatant from aMoC1 cell cultures in serum-free medium at $24 \mathrm{~h}$ after plating and centrifuging for $10 \mathrm{~min}$ at 3,000 x g; aMoC1-CM was used at $50 \%$ concentration in all experiments.

Generation of KRasV12-expressing aMoCl cells.FLAG-tagged human KRasV12 was PCR-amplified from a human colorectal cancer cell line, SW620, that carries the KRasV12 mutation, and was subcloned into the EcoRI/NotI sites of pLVSIN-EF1 $\alpha$ IRES-ZsGreen1 vector (Takara Bio, Shiga, Japan). The empty vector was used as a control. Lentivirus was produced as previously described (25), and aMoC1 cells were treated with virus-containing supernatants for 12-24 h. ZsGreen-expressing cells were sorted using an SH800 flow cytometer (Sony, Tokyo, Japan), and were used as mock-transfected aMoC1 cells (Mock-aMoC1 cells) and KRasV12-expressing aMoC1 cells (KRasV12-aMoC1 cells).

Western blotting. Western blotting was performed as previously described (26). Briefly, cells were lysed in a buffer containing $50 \mathrm{mM}$ Tris- $\mathrm{HCl}(\mathrm{pH} 8.0), 5 \mathrm{mM}$ EDTA (pH 8.0), 5 mM EGTA (pH 8.0), 1\% Triton X-100, $1 \mathrm{mM} \mathrm{Na}_{3} \mathrm{VO}_{4}$, $20 \mathrm{mM}$ sodium pyrophosphate, and Roche complete proteaseinhibitor cocktail (Roche, Basel, Switzerland). Proteins were quantified using a Bio-Rad DC protein assay kit (Bio-Rad, Hercules, CA, USA) and then separated using sodium dodecyl sulfate-polyacrylamide gel electrophoresis and transferred onto nitrocellulose membranes (Wako Pure Chemical Industries, Osaka, Japan). The membranes were blocked with $0.5 \%$ skim milk and then probed with primary and secondary antibodies, and the stained protein bands were detected using Western Lightning ECL Pro (PerkinElmer, Waltham, MA, USA) and visualized using a LAS-3000 mini luminescence imager (Fujifilm, Tokyo, Japan). The primary antibodies used were against these targets: c-Myc, GSK-3 $\beta$ phospho-Ser9,
ERK p44/42 phospho-Thr202/Tyr204, Akt1 phospho-Ser473, p38 MAPK phospho-Thr180/Tyr182, p70 S6 Kinase (S6K) phospho-Thr389, Stat3 $\alpha$ phospho-Tyr705, and Yap phosphoSer127 (Cell Signaling Technology, Danvers, MA, USA); JNK phospho-Thr183/Tyr185 (Promega, Madison, WI, USA); $\alpha$-smooth muscle actin ( $\alpha$-SMA) and vimentin (Sigma, St. Louis, MO, USA); actin (Santa Cruz Biotechnology, Dallas, TX, USA); Ras (Merck Millipore, Darmstadt, Germany); tubulin (Thermo Fisher Scientific); CD44 (Bethyl Laboratories, Montgomery, TX, USA); Lgr5 (Abgent, San Diego, CA, USA); and p97/VCP (GeneTex, Irvine, CA, USA). The secondary HRP-conjugated antibodies used were goat anti-mouse IgG (R\&D Systems, Minneapolis, MN, USA), goat anti-rabbit IgG (Cayman Chemical Co., Ann Arbor, MI, USA), and donkey anti-goat IgG (Bethyl Laboratories) antibodies.

Cell proliferation assay. At $24 \mathrm{~h}$ (day 1) and $72 \mathrm{~h}$ (day 3) after cell seeding, cell proliferation was analyzed by using a Cell Counting Kit-8 (Dojindo Laboratories, Kumamoto, Japan) as per the manufacturer's instructions, and the proliferative rate was calculated as the day 3/day 1 rate. For IMFs, CM was added to the culture medium at $24 \mathrm{~h}$ after seeding.

Boyden chamber assay. Boyden chamber migration assays were performed using transwell chambers (Corning, Corning, NY, USA) as previously described (27). For invasion assays, $8-\mu \mathrm{m}$-pore-size polycarbonate membranes were coated with $10 \%$ Matrigel, and then $800 \mu \mathrm{l}$ of serum-free DMEM was added in the lower chamber and $1 \times 10^{4} \mathrm{aMoC} 1$ cells or $4 \times 10^{4}$ IMFs in $200 \mu \mathrm{l}$ of serum-free DMEM were added in the upper chamber. After $2 \mathrm{~h}, 5 \% \mathrm{FBS}$ (for aMoC1 cells) or $50 \% \mathrm{CM}$ (for IMFs) was added to the lower chamber and the cells were cultured for $6 \mathrm{~h}$ (aMoC1 cells) or $12 \mathrm{~h}$ (IMFs). Membranes were fixed with methanol for $15 \mathrm{~min}$ and stained with Giemsa (Muto Pure Chemicals, Tokyo, Japan). After washing the membranes with Milli-Q water, non-migrated cells were wiped off with a cotton-swab, and then the migrated cells were counted in 3-5 randomly selected x 200 fields under a light microscope and averaged.

Soft-agar colony-formation assay. Into each well of 6-well plates, $2.5 \mathrm{ml}$ of $0.75 \%$ agarose in DMEM containing $2.8 \%$ $\mathrm{NaHCO}_{3}, 10 \% \mathrm{FBS}$, and $1 \%$ AA was poured and allowed to solidify at $4^{\circ} \mathrm{C}$ for $8 \mathrm{~min}$. After solidification, 1,000 cells were suspended in $1 \mathrm{ml}$ of $0.36 \%$ agarose in the same medium and then plated on top of the base layer. The cells were cultured for 2 weeks, and colonies were stained with crystal violet.

Wound-healing migration assay. Cells were cultured in 35-mm dishes as confluent monolayers. The monolayers were cultured in serum-free medium overnight, wounded in a line across the well by using a $200-\mu 1$ pipette tip, and incubated in fresh serum-free medium containing $\mathrm{CM}$ and/or cytokines (10 ng/ml) for $6 \mathrm{~h}$ (LmcMF cells) or $12 \mathrm{~h}$ (primary IMFs). Cells were pretreated with inhibitors for $30 \mathrm{~min}$. Wound areas were measured using ImageJ software (National Institutes of Health, Bethesda, MD, USA).

Quantitative real-time PCR. Total RNA was extracted from cells by using TRIzol (Thermo Fisher Scientific) as per the 


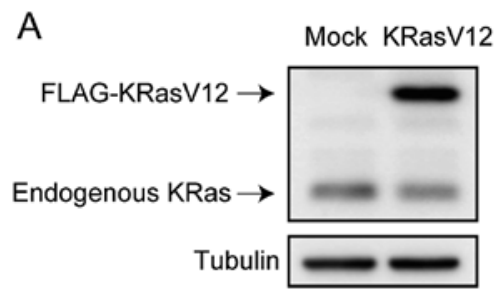

C

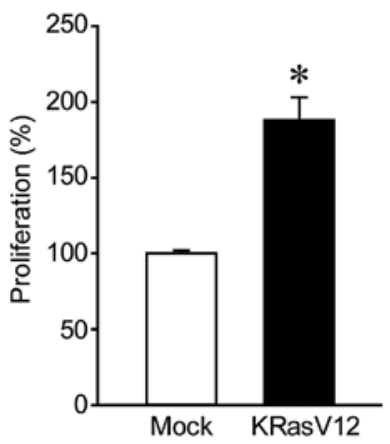

E

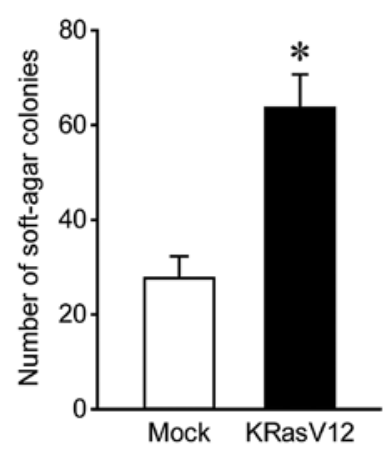

B
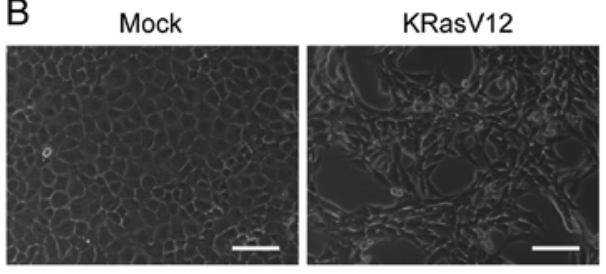

D

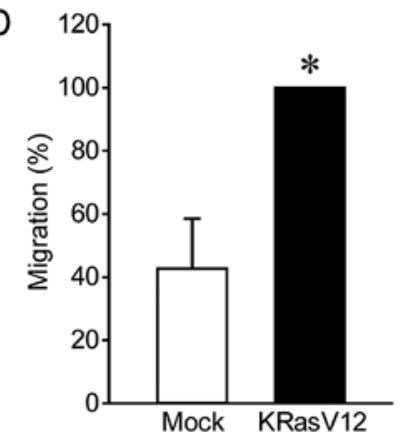

F

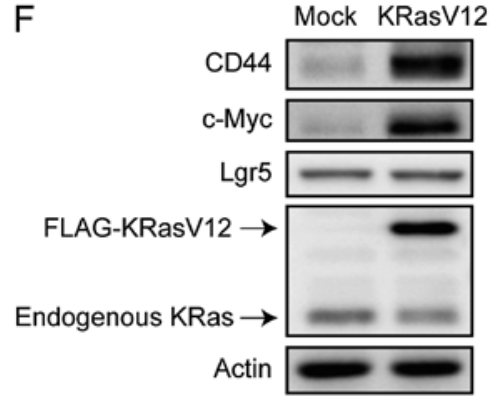

Figure 1. KRasV12 overexpression promotes tumorigenesis of aMoC1 cells. (A) Western blot analysis of KRasV12 overexpression. Tubulin was used as a loading control. Representative images from 3 independent experiments are shown. (B) Representative phase-contrast images of Mock-aMoC1 (Mock) and KRasV12-aMoC1 (KRasV12) cells. Scale bar, $100 \mu \mathrm{m}$. (C) Effect of KRasV12 overexpression on the proliferation of aMoC1 cells. The proliferation rate of Mock-aMoC1 cells was defined as $100 \%$. $\mathrm{n}=6$ in duplicate. (D) Effect of KRasV12 overexpression on the migration of aMoC1 cells. Cell migration was measured using Boyden chamber assays. The migration rate of $\mathrm{KRasV} 12-\mathrm{aMoC} 1$ cells was defined as $100 \%$. $\mathrm{n}=3$. (E) Effect of KRasV12 overexpression on anchorage-independent growth of aMoC1 cells. $n=6$ in duplicate. (F) Western blot analysis of the expression of stem cell markers (CD44, c-Myc, and Lgr5) in Mock-aMoC1 and KRasV12-aMoC1 cells. Actin was used as a loading control. Representative images from 3 independent experiments are shown. Quantitative results are expressed as means \pm SEM. ${ }^{*} \mathrm{P}<0.05$ vs. Mock.

manufacturer's protocol. RNA concentration was adjusted to $0.5 \mu \mathrm{g} / \mu \mathrm{l}$, and quantitative real-time PCR was performed using a QuantiTect Reverse Transcription kit, a QuantiTect SYBR I kit (Qiagen, Hilden, Germany), and CFX96 Touch ${ }^{\text {TM }}$ Real-time PCR Detection System (Bio-Rad), following manufacturer protocols. The $\Delta \Delta \mathrm{Cq}$ method was used for quantification (28). The specific primers used for mouse epidermal growth factor (EGF), HB-EGF, insulin-like growth factor-1 (IGF-1), IL-6, TGF- $\beta 1$, tumor necrosis factor- $\alpha$ (TNF- $\alpha$ ), and $\beta$-actin were the following: EGF, forward CCCAGGCAACGTATCAAAGT, reverse GGTCATACCCAGGAAAGCAA; HB-EGF, forward GACCCATGCCTCAGGAAATA, reverse TGAGAAGTCC CACGATGACA; IGF-1, forward TTCAGTTCGTGTGTGG ACCGAG, reverse TCCACAATGCCTGTCTGAGGTG; IL-6, forward CACGGCCTTCCCTACTTCAC, reverse TGCAAGTGCATCATCGTTGT; TGF- $\beta 1$, forward TTGC TTCAGCTCCACAGAGA, reverse TGGTTGTAGAGGGC AAGGAC; TNF- $\alpha$, forward AGACCCTCACACTCAGATC ATCTTC, reverse TTGCTACGACGTGGGCTACA; and $\beta$-actin, forward GATTACTGCTCTGGCTCCTAGC, reverse GACTCATCGTACTCCTGCTTGC.
Reagents. The cytokines used were: HB-EGF (Sigma); EGF, IL-6 (PeproTech, Rocky Hill, NJ, USA); TGF- $\beta 1$ (AMBiS, Okinawa, Japan); TNF- $\alpha$ (Wako Pure Chemical Industries); and IGF-1 (ProSpec-Tany TechnoGene Ltd., Rehovot, Israel). The inhibitors used were dacomitinib (Wako Pure Chemical Industries), FR180204 (Sigma), and SP600125 (Cayman Chemical Co.).

Statistical analysis. Results are expressed as means \pm SEM. Student's t-test was used for comparison between two groups. Groups of three or more were compared using one-way analysis of variance, followed by Tukey's test. In all analyses, $\mathrm{P}<0.05$ was considered statistically significant.

\section{Results}

KRasV12 overexpression converts aMoCl cells into tumor cells. We first analyzed the oncogenic effects of KRasV12 overexpression in aMoC1 cells. KRasV12 was expressed at higher levels in KRasV12-overexpressing aMoC1 cells (KRasV12-aMoC1 cells) than in control cells transfected with 

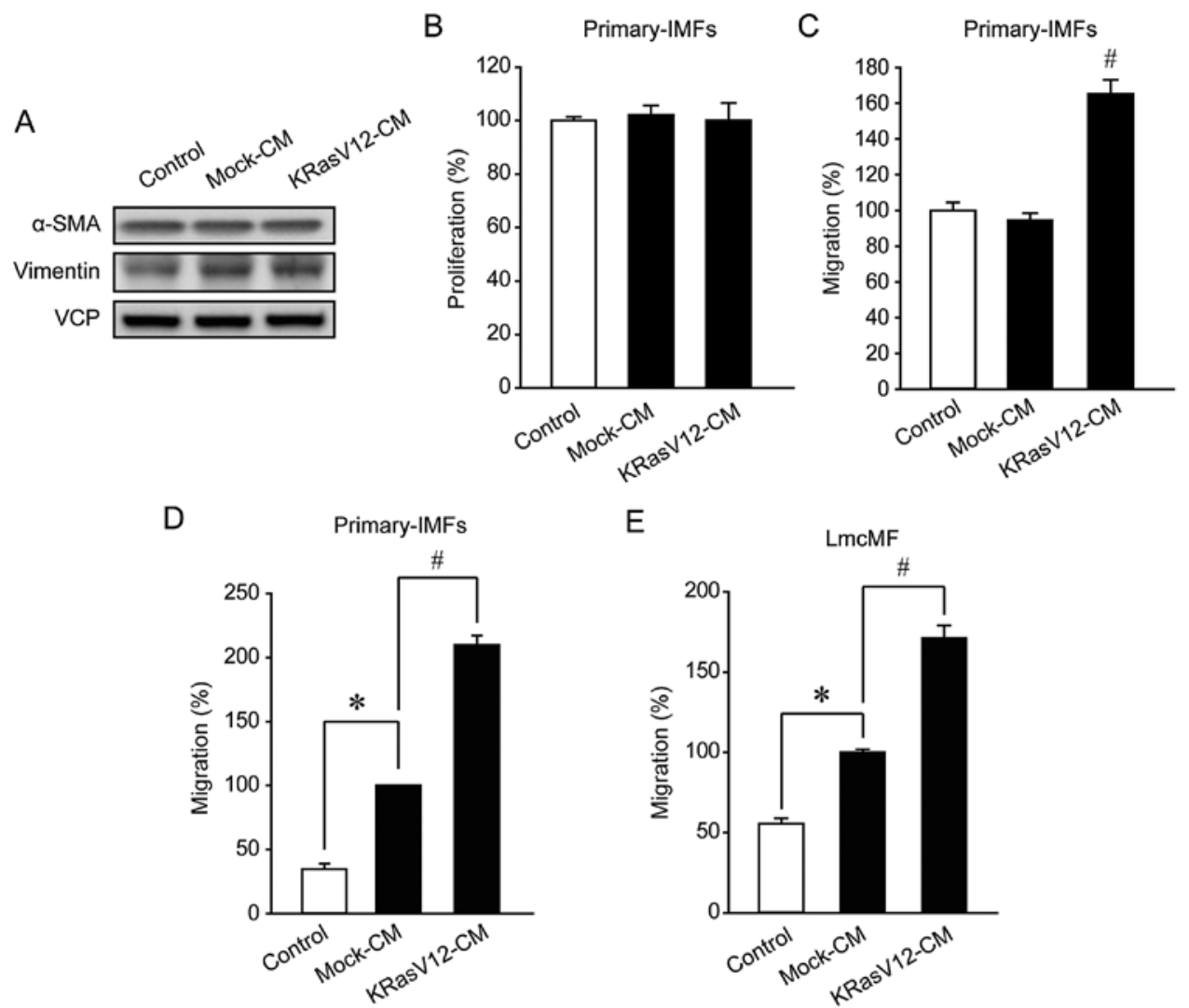

Figure 2. Conditioned medium (CM) of KRasV12-aMoC1 cells promotes IMF migration. (A) Western blot analysis of the expression of myofibroblast markers ( $\alpha$-SMA and vimentin) in primary IMFs that were either not treated or treated with Mock-CM or KRasV12-CM for 5 days. VCP was used as a loading control. Representative images from 2 independent experiments are shown. $\mathrm{n}=4$ in duplicate. (B) Effect of KRasV12-CM on the proliferation of primary IMFs. The proliferation rate of non-treated primary IMFs was defined as $100 \%$. $n=6$ in duplicate. Effect of KRasV12-CM on primary-IMF migration. Cell migration was measured using wound-healing assays (C) and Boyden chamber assays (D). The migration rates of non-treated primary IMFs (C) or Mock-CM-treated primary IMFs (D) were defined as $100 \%$. $\mathrm{n}=12$ in duplicate (C) or 3 (D). (E) Effect of KRasV12-CM on LmcMF cell migration, measured using wound-healing assays. The migration rate of Mock-CM-treated LmcMF cells was defined as $100 \% . \mathrm{n}=18-20$ in duplicate. Quantitative results are expressed as means \pm SEM. ${ }^{*} \mathrm{P}<0.05$ vs. non-treated cells; ${ }^{~} \mathrm{P}<0.05$ vs. Mock-CM-treated cells.

the empty vector (Mock-aMoC1 cells) (Fig. 1A). KRasV12 overexpression altered the morphology of aMoC1 cells, leading to the formation of atypically shaped cells (Fig. 1B). We further confirmed that KRasV12 overexpression enhanced the proliferation, migration, and anchorage-independent growth of aMoC1 cells (Fig. 1C-E). Since KRas mutations have been reported to enhance stemness $(29,30)$, we examined the expression of the cancer stem cell markers, CD44, c-Myc, and Lgr5. KRasV12 overexpression increased the expression of CD44 and c-Myc but not Lgr5 (Fig. 1F). These results suggest that KRasV12 overexpression converts colorectal epithelial cells into tumor cells.

KRasV12-aMoC1 CM promotes IMF migration. To investigate how KRasV12 overexpression in epithelial cells affects the functions of IMFs, we treated IMFs with the CM from Mock-aMoC1 cells (Mock-CM) or KRasV12-aMoC1 cells (KRasV12-CM) and then examined IMF differentiation, proliferation, and migration. IMF differentiation was evaluated based on the expression level of myofibroblast markers ( $\alpha$-SMA and vimentin) as previously described (23). In primary IMFs, KRasV12-CM exerted little effect on differentiation and proliferation (Fig. 2A and B). By contrast, primaryIMF migration was promoted by KRasV12-CM compared with Mock-CM (Fig. 2C). Furthermore, in Boyden chamber assays, IMF migration was weakly promoted by Mock-CM but strongly promoted by KRasV12-CM (Fig. 2D). Similarly, in the IMF cell line LmcMF, Mock-CM and KRasV12-CM weakly and strongly promoted migration, respectively (Fig. 2E). These results indicate that KRas-mutated colorectal cancer cells promote IMF migration but do not influence IMF differentiation and proliferation.

HB-EGF is upregulated in KRasV12-aMoCl cells and mediates IMF migration. To clarify the mechanisms of KRasV12-CM-mediated promotion of IMF migration, we compared cytokine expression levels in Mock-aMoC1 and KRasV12-aMoC1 cells: Quantitative real-time PCR analyses revealed that IL- 6 , TNF- $\alpha$, TGF- $\beta 1$, EGF, and HB-EGF were expressed at higher levels in KRasV12-aMoC1 cells than in Mock-aMoC1 cells, whereas IGF-1 was expressed at a lower level in KRasV12-aMoC1 cells (Fig. 3A). Among these cytokines, HB-EGF markedly promoted the migration of primary IMFs and LmcMF cells (Fig. 3B and C). We further confirmed that HB-EGF promoted IMF migration by performing Boyden chamber assays (Fig. 3D). These results imply that KRas-mutated cancer cells promote IMF migration through HB-EGF upregulation. 

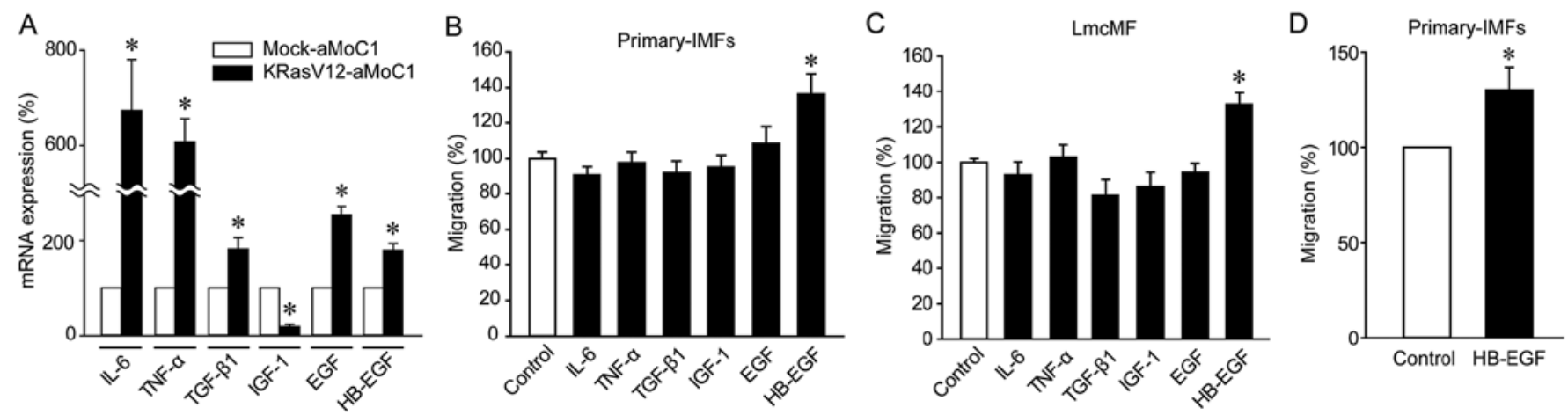

Figure 3. HB-EGF is upregulated in KRasV12-aMoC1 cells and promotes IMF migration. (A) Quantitative real-time PCR analysis of the mRNA expression of the indicated cytokines in Mock-aMoC1 (control) and KRasV12-aMoC1 cells. The mRNA expression of each cytokine in control cells was defined as 100\%. $\mathrm{n}=5$. Effects of indicated cytokines $(10 \mathrm{ng} / \mathrm{ml})$ on the migration of primary IMFs (B) or LmcMF cells (C). Cell migration was measured using wound-healing assays. Non-treated primary IMFs (B) or Mock-CM-treated LmcMF cells (C) were used as the control; the migration rate of control was defined as $100 \%$. $\mathrm{n}=6$ in duplicate (B) or 4-12 in duplicate (C). (D) Effect of HB-EGF (10 ng/ml) on primary-IMF migration. Cell migration was measured using Boyden chamber assays. Mock-CM-treated primary IMFs were used as the control; the migration rate of control was defined as $100 \%$. $n=3$. Quantitative results are expressed as means \pm SEM. ${ }^{*} \mathrm{P}<0.05$ vs. control.
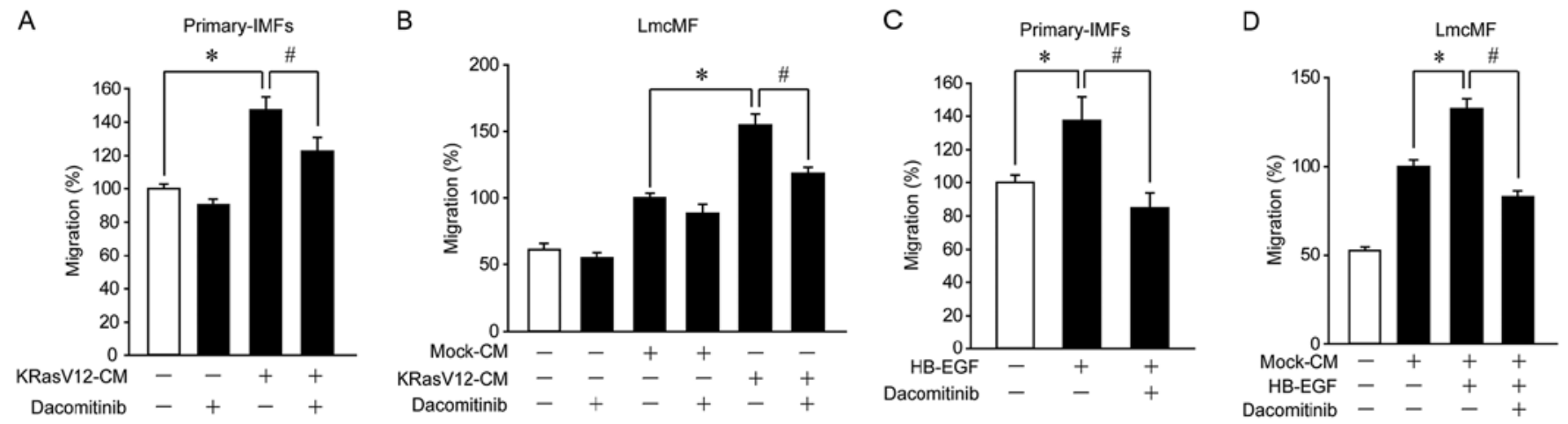

Figure 4. KRasV12-aMoC1 cell CM and HB-EGF promote IMF migration through activation of HB-EGF receptors. Effect of dacomitinib (1 $\mu \mathrm{M})$ pretreatment (30 min) on KRasV12-CM-induced migration of primary IMFs (A) and LmcMF cells (B). Cell migration was measured using wound-healing assays. Non-treated primary IMFs (A) or Mock-CM-treated LmcMF cells (B) were used as the control; the migration rate of control was defined as 100\%. $\mathrm{n}=14-20$ in duplicate (A) and 4 in duplicate (B). Effect of dacomitinib (1 $\mu \mathrm{M})$ pretreatment (30 min) on HB-EGF $(10 \mathrm{ng} / \mathrm{ml})$ induced migration of primary IMFs (C) and LmcMF cells (D). Cell migration was measured using wound-healing assays. Non-treated primary IMFs (C) or Mock-CM-treated LmcMF cells (D) were used as the control; the migration rate of control was defined as $100 \%$. $\mathrm{n}=6$ in duplicate. Quantitative results are expressed as means $\pm \mathrm{SEM}$. ${ }^{\mathrm{P}}<0.05$ vs. control; ${ }^{\#} \mathrm{P}<0.05$ vs. KRasV12-CM- or HB-EGF-treated cells.

KRasV12-aMoCl cells promote IMF migration through activation of $H B-E G F$ receptors. Since HB-EGF is recognized as a ligand of ErbB1 and ErbB4 (31), we analyzed the involvement of these receptors in KRasV12-CM-induced IMF migration. In primary IMFs and LmcMF cells, KRasV12-CM-induced migration was suppressed by dacomitinib, an inhibitor of ErbB1, ErbB2, and ErbB4 (Fig. 4A and B). Furthermore, dacomitinib suppressed HB-EGF-induced migration in primary IMFs and LmcMF cells (Fig. 4C and D). These results suggest that KRas-mutated cancer cells promote IMF migration through activation of HB-EGF receptors.

HB-EGF promotes IMF migration through activation of ERKI/2 and JNK signals. Lastly, we investigated the mechanisms underlying HB-EGF-mediated promotion of IMF migration by examining the effects of KRasV12-CM and HB-EGF on the activation of various cellular signaling pathways. Western blot analyses revealed that whereas KRasV12-CM increased the phosphorylation of Stat3, p38 MAPK, ERK1/2, and JNK in LmcMF cells (Fig. 5), HB-EGF increased the phosphorylation of GSK-3 $\beta$, S6K, Akt, ERK1/2, and JNK in these cells (Fig. 6). Since ERK1/2 and JNK were activated by both KRasV12-CM and HB-EGF, we hypothesized that ERK1/2 and JNK are involved in KRasV12CM-induced IMF migration. To test this, we measured KRasV12-CM-induced IMF migration after treatment with the ERK1/2 inhibitor FR180204 or the JNK inhibitor SP600125: Both compounds strongly inhibited KRasV12-CM-induced migration in LmcMF cells, but partially suppressed basal and Mock-CM-induced migration (Fig. 7A and B). Moreover, our results confirmed that the activation of ERK1/2 and JNK by KRasV12-CM was blocked by dacomitinib in LmcMF cells (Fig. 7C-E). These results suggest that HB-EGF secreted from KRasV12-mutated cancer cells promotes IMF migration through activation of ERK1/2 and JNK signals (Fig. 8).

\section{Discussion}

In colorectal cancer, KRas mutations occur frequently and are known to play key roles in carcinogenesis. However, 
A
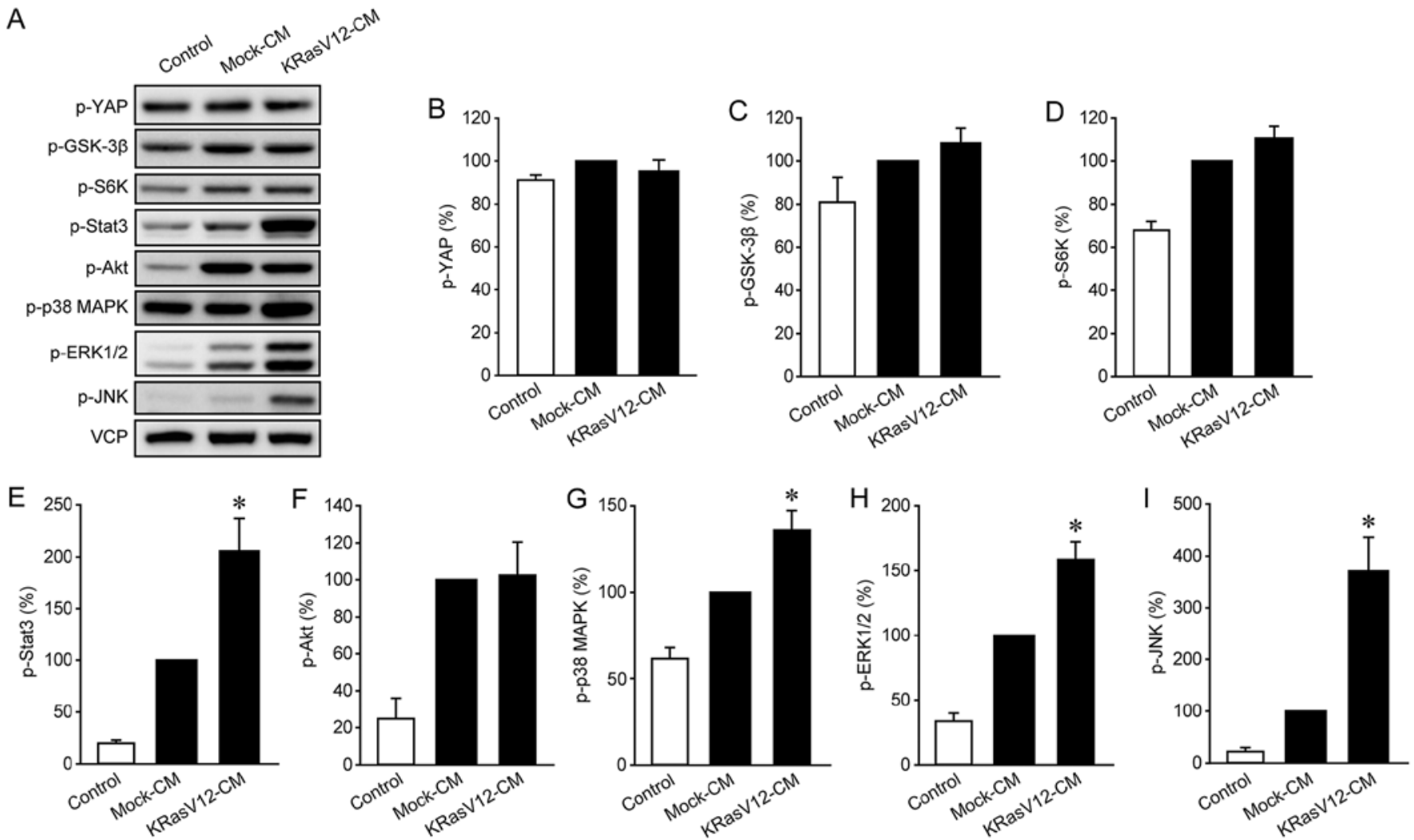

Figure 5. KRasV12-aMoC1 cell CM activates intracellular signaling in IMFs. Western blotting was performed using the indicated antibodies to analyze the effects of Mock-CM and KRasV12-CM treatment (15 min) on intracellular signaling in LmcMF cells. VCP was used as a loading control. Representative images are shown (A). The expression level in Mock-CM-treated LmcMF cells was defined as 100\%. Quantitative results are expressed as means \pm SEM (B-I). ${ }^{*} \mathrm{P}<0.05$ vs. Mock-CM-treated LmcMF cells. $\mathrm{n}=5$ (B, C, E and I), 6 (D), 7 (F and G), and 10 (H).
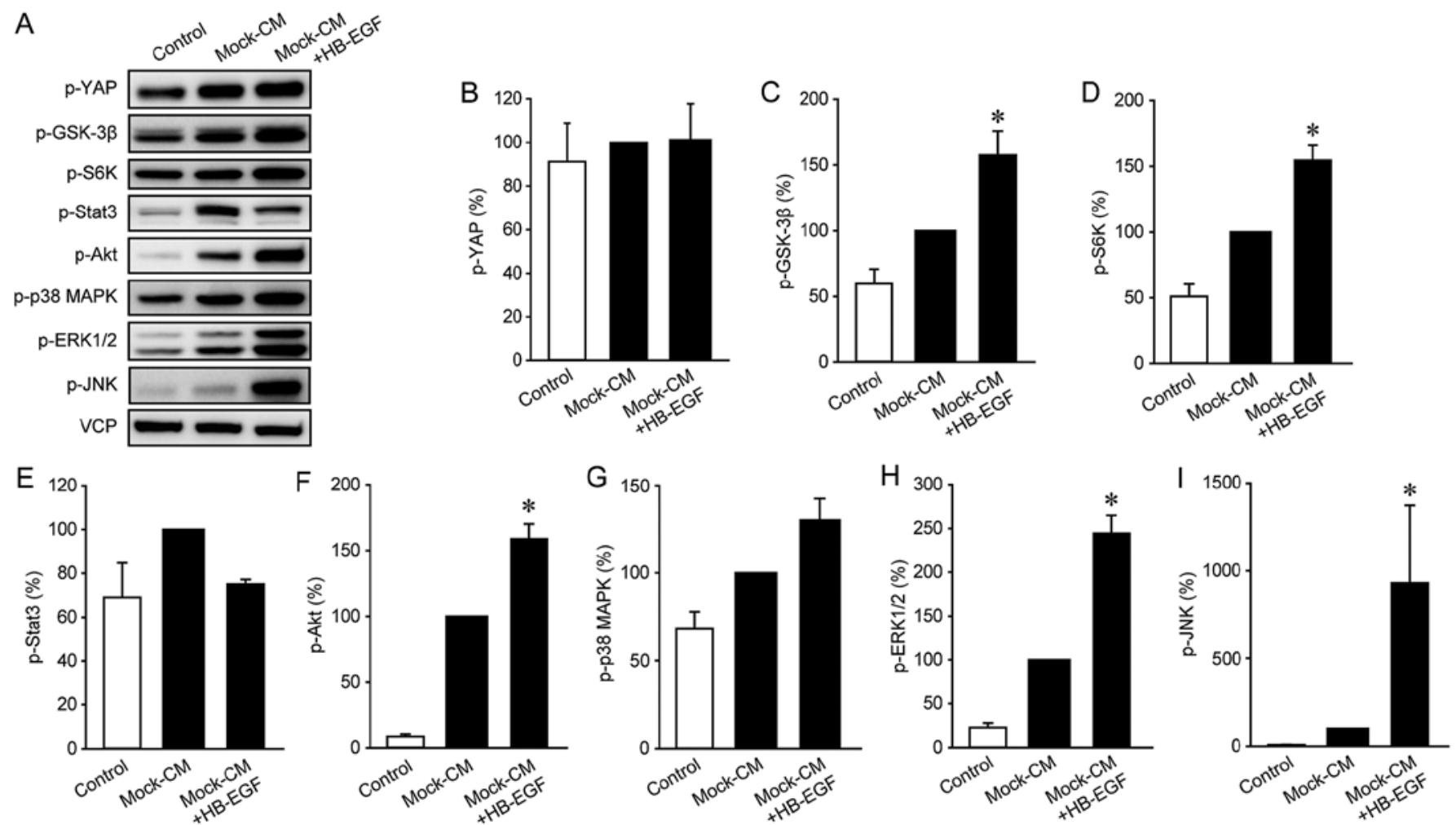

Figure 6. HB-EGF activates intracellular signaling in IMFs. Western blotting was performed using the indicated antibodies to analyze the effect of 15 -min treatment with Mock-CM added with or without HB-EGF $(10 \mathrm{ng} / \mathrm{ml})$ on intracellular signaling in LmcMF cells. VCP was used as a loading control. Representative images are shown (A). The expression level in Mock-CM-treated LmcMF cells was defined as 100\%. Quantitative results are expressed as means \pm SEM (B-I). ${ }^{*} \mathrm{P}<0.05$ vs. Mock-CM-treated LmcMF cells. $\mathrm{n}=3$ (B-E and $\left.\mathrm{I}\right), 4$ ( $\mathrm{F}$ and $\left.\mathrm{G}\right)$, and $5(\mathrm{H})$. 
A

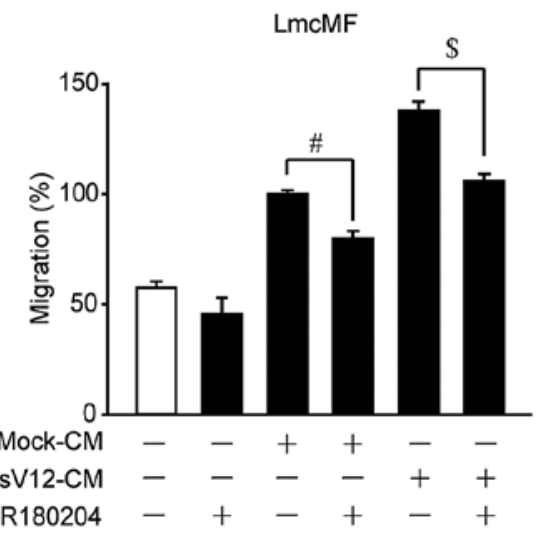

B

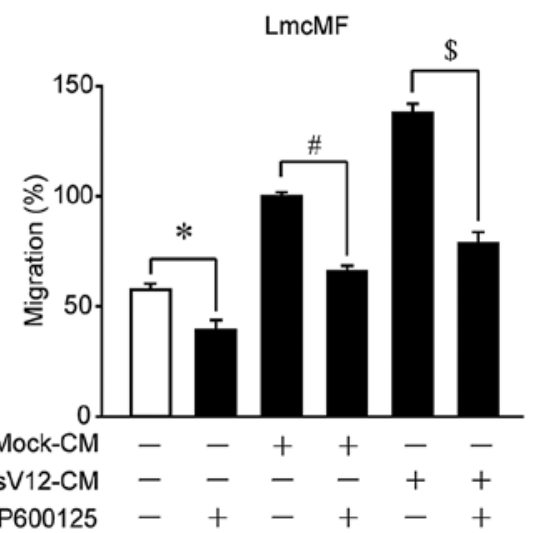

C
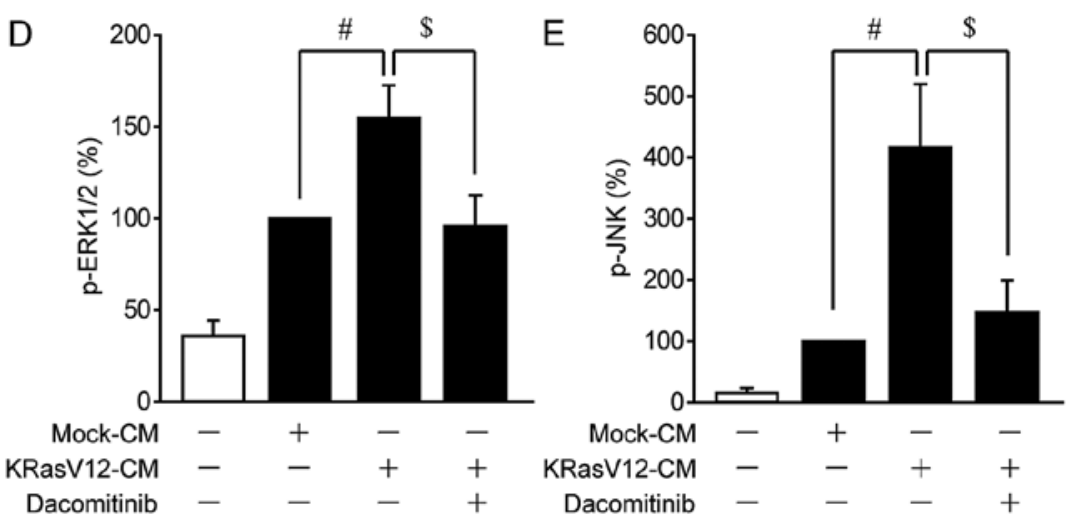

Figure 7. KRasV12-aMoC1 cell CM promotes IMF migration by activating ERK1/2 and JNK through HB-EGF receptors. Effect of 30-min pretreatment with FR180204 (ERK1/2 inhibitor; $1 \mu \mathrm{M}$ ) (A) or SP600125 (JNK inhibitor; $1 \mu \mathrm{M})$ (B) on KRasV12-CM-induced LmcMF cell migration. Cell migration was measured using wound-healing assays. The migration rate of Mock-CM-treated LmcMF cells was defined as 100\%. $\mathrm{n}=6-10$ in duplicate. Western blotting was performed to analyze the effect of dacomitinib $(1 \mu \mathrm{M})$ pretreatment (30 min) on KRasV12-CM-stimulated phosphorylation of ERK1/2 (C and D) and JNK (C and E) in LmcMF cells. VCP was used as a loading control. Representative images are shown (C). The expression level in Mock-CM-treated LmcMF cells was defined as $100 \%$. $n=7$ (D) and 3 (E). Quantitative results are expressed as means \pm SEM (D and E). ${ }^{*} \mathrm{P}<0.05$ vs. non-treated LmcMF cells; ${ }^{*} \mathrm{P}<0.05$ vs. Mock-CM-treated LmcMF cells; ${ }^{\mathrm{P}}<0.05$ vs. KRasV12-CM-treated LmcMF cells.

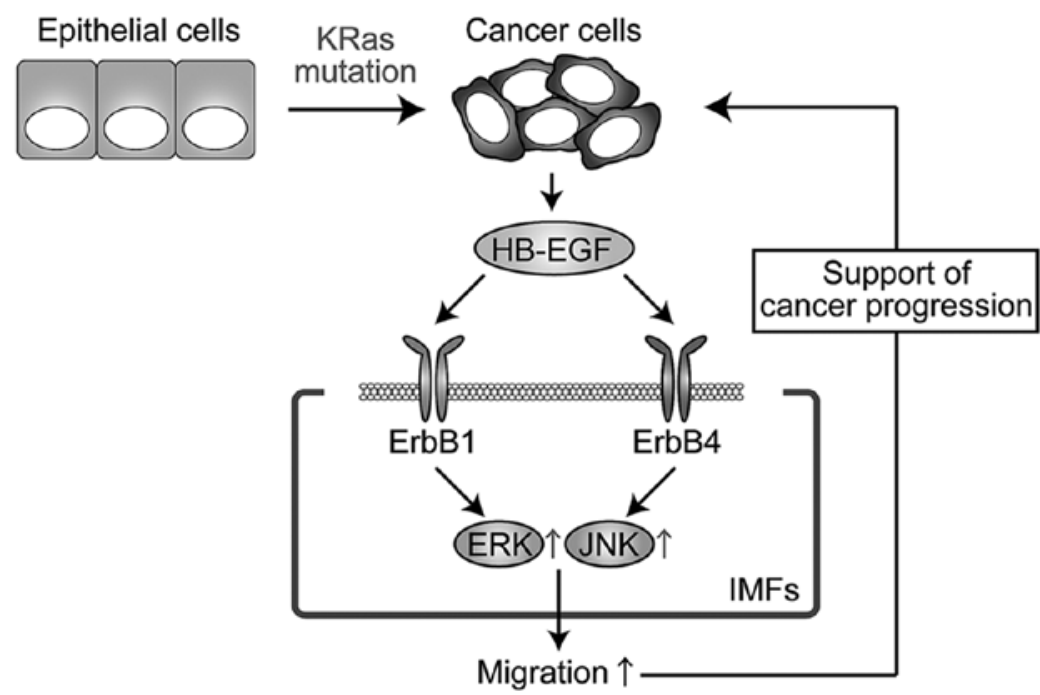

Figure 8. IMF migration promoted by KRas-mutated cancer cells: a model. KRas mutation induces the transformation of colorectal epithelial cells and upregulates HB-EGF expression. HB-EGF secreted from KRas-mutated cells promotes IMF migration by activating ERK1/2 and JNK signaling through ErbB1 and ErbB4, which, in turn, could support cancer progression.

the molecular mechanisms by which KRas mutations in epithelial cells affect the functions of myofibroblasts have remained unclear. We demonstrated here, for the first time, that HB-EGF secreted from KRas-mutated colorectal cancer cells promotes IMF migration through the activation of ERK and JNK signals.

We used KRasV12-aMoC1 cells as a model of colorectal cancer cells for investigating the functions of KRas-mutated 
colorectal cancer cells. Consistent with previous reports $(3,6,29)$, KRasV12 expression transformed aMoC1 cells, which was demonstrated by our results showing that KRasV12 promoted aMoCl cell proliferation, migration, and anchorage-independent growth (Fig. 1). Moreover, KRasV12 upregulated the expression of CD44 and c-Myc, which are involved in the stemness of colorectal cancer cells $(32,33)$. Our findings suggest that KRasV12 overexpression converts colorectal epithelial cells into tumor cells.

KRasV12 overexpression in aMoC1 cells increased the expression of TGF- $\beta 1$, EGF, and TNF- $\alpha$ (Fig. 3A), which are recognized as major regulators of myofibroblast differentiation and proliferation (34-36), but KRasV12-CM exerted little effect on the differentiation and proliferation of IMFs (Fig. 2A and B). However, KRasV12 overexpression decreased the levels of IGF-1, which is also involved in the differentiation and proliferation of myofibroblasts (34-36). Based on these results, we propose that IGF-1 downregulation might suppress the IMF proliferation and differentiation induced by the other cytokines examined here. In contrast to these results, we found that KRasV12-CM increased IMF migration and chemotaxis as compared with Mock-CM (Fig. 2C-E). These findings suggest that KRas-mutated cancer cells attract IMFs mainly through migration and thus generate a cancer microenvironment.

HB-EGF is an EGF-family molecule that regulates cell proliferation and differentiation (31). Moreover, in cancer progression, HB-EGF promotes tumor cell growth/survival and angiogenesis $(31,37)$, whereas, HB-EGF promotes the migration of epithelial cells and fibroblasts (38-40). Herein, HB-EGF expression was upregulated in KRasV12-aMoC1 cells, and HB-EGF enhanced IMF migration (Fig. 3). Moreover, KRasV12-CM- and HB-EGF-induced IMF migration was inhibited by dacomitinib (Fig. 4). These results suggest that KRasV12-aMoC1 cells promoted IMF migration through HB-EGF/ErbB signaling. However, dacomitinib did not completely inhibit KRasV12-CM-induced IMF migration. Thus, several other factors present in KRasV12-CM besides HB-EGF might also be involved in promoting IMF migration, such as PDGF, which is recognized to promote myofibroblast migration (41). Nevertheless, our results support the conclusion that HB-EGF is one of the major factors in KRasV12-CM that promotes IMF migration.

The results of this study showed that HB-EGF and KRasV12-CM activate ERK1/2 and JNK signals (Figs. 5 and 6). ERK and JNK signaling pathways are widely recognized to be involved in cell migration $(42,43)$. Accordingly, we found that inhibition of ERK1/2 and JNK suppressed KRasV12-CM-induced IMF migration (Fig. 7A and B). Given that KRasV12-CM-induced activation of ERK1/2 and JNK was inhibited by dacomitinib (Fig. 7C-E), our results indicate that the activation of these signals by KRasV12-CM is mediated by HB-EGF receptors.

Myofibroblasts are components of the cancer microenvironment and they promote cancer progression (44). The findings of this study suggest that KRas-mutated colorectal cancer cells enhance IMF migration and attract IMFs toward the cancer cells, which might contribute to cancer progression. Since these mechanisms of IMF migration might be essential for the generation or maintenance of the cancer microenvironment, HB-EGF, due to its ability to regulate the cancer microenvironment, could potentially emerge as a novel therapeutic target in the treatment of patients with KRas-mutated colorectal cancer.

In conclusion, our results suggest that HB-EGF secreted from KRas-mutated colorectal cancer cells promotes IMF migration through ErbB receptors, ERK1/2, and JNK and thereby generates a tumor microenvironment that favors cancer progression. Further investigation into the interaction between KRas-mutated cells and IMFs could contribute to research on the cancer microenvironment and the development of new therapies targeting IMFs.

\section{Acknowledgements}

This work was partly supported by a Grant-in-Aid for Scientific Research from the Japanese Ministry of Education, Culture, Sports, Science and Technology (grant nos.: 15K14855, K.S.; 14J06412, H.K.). We thank Dr Mamoru Totsuka (Tokyo University) for providing the aMoC1 cells.

\section{References}

1. Torre LA, Bray F, Siegel RL, Ferlay J, Lortet-Tieulent J and Jemal A: Global cancer statistics, 2012. CA Cancer J Clin 65: 87-108, 2015.

2. Chang YY, Lin PC, Lin HH, Lin JK, Chen WS, Jiang JK, Yang SH, Liang WY and Chang SC: Mutation spectra of RAS gene family in colorectal cancer. Am J Surg 212: 537-544.e3, 2016.

3. Pollock CB, Shirasawa S, Sasazuki T, Kolch W and Dhillon AS: Oncogenic K-RAS is required to maintain changes in cytoskeletal organization, adhesion, and motility in colon cancer cells. Cancer Res 65: 1244-1250, 2005.

4. Bäumer S, Bäumer N, Appel N, Terheyden L, Fremerey J, Schelhaas S, Wardelmann E, Buchholz F, Berdel WE and Müller-Tidow C: Antibody-mediated delivery of anti-KRASsiRNA in vivo overcomes therapy resistance in colon cancer. Clin Cancer Res 21: 1383-1394, 2015.

5. Pereira AA, Rego JF, Morris V, Overman MJ, Eng C, Garrett CR, Boutin AT, Ferrarotto R, Lee M, Jiang ZQ, et al: Association between KRAS mutation and lung metastasis in advanced colorectal cancer. Br J Cancer 112: 424-428, 2015.

6. Feng Y, Bommer GT, Zhao J, Green M, Sands E, Zhai Y, Brown K, Burberry A, Cho KR and Fearon ER: Mutant KRAS promotes hyperplasia and alters differentiation in the colon epithelium but does not expand the presumptive stem cell pool. Gastroenterology 141: 11003-1013.e1-10, 2011.

7. Yoshida M, Taguchi A, Kawana K, Adachi K, Kawata A, Ogishima J, Nakamura H, Fujimoto A, Sato M, Inoue T, et al: Modification of the tumor microenvironment in KRAS or c-MYC-induced ovarian cancer-associated peritonitis. PLoS One 11: e0160330, 2016.

8. Sparmann A and Bar-Sagi D: Ras-induced interleukin-8 expression plays a critical role in tumor growth and angiogenesis. Cancer Cell 6: 447-458, 2004.

9. Pylayeva-Gupta Y, Lee KE, Hajdu CH, Miller G and Bar-Sagi D: Oncogenic Kras-induced GM-CSF production promotes the development of pancreatic neoplasia. Cancer Cell 21: 836-847, 2012.

10. Quante M, Varga J, Wang TC and Greten FR: The gastrointestinal tumor microenvironment. Gastroenterology 145: 63-78, 2013.

11. Augsten $M$, Hägglöf $C$, Peña $C$ and Ostman A: A digest on the role of the tumor microenvironment in gastrointestinal cancers. Cancer Microenviron 3: 167-176, 2010.

12. Hanahan D and Coussens LM: Accessories to the crime: Functions of cells recruited to the tumor microenvironment. Cancer Cell 21: 309-322, 2012.

13. Tahara E: Abnormal growth factor/cytokine network in gastric cancer. Cancer Microenviron 1: 85-91, 2008.

14. Avgustinova A, Iravani M, Robertson D, Fearns A, Gao Q, Klingbeil P, Hanby AM, Speirs V, Sahai E, Calvo F, et al: Tumour cell-derived Wnt7a recruits and activates fibroblasts to promote tumour aggressiveness. Nat Commun 7: 10305, 2016. 
15. Chivukula RR, Shi G, Acharya A, Mills EW, Zeitels LR, Anandam JL, Abdelnaby AA, Balch GC, Mansour JC, Yopp AC, et al: An essential mesenchymal function for miR-143/145 in intestinal epithelial regeneration. Cell 157: 1104-1116, 2014.

16. Powell DW, Adegboyega PA, Di Mari JF and Mifflin RC: Epithelial cells and their neighbors I. Role of intestinal myofibroblasts in development, repair, and cancer. Am J Physiol Gastrointest Liver Physiol 289: G2-G7, 2005.

17. Vermeulen L, De Sousa E Melo F, van der Heijden M, Cameron K, de Jong JH, Borovski T, Tuynman JB, Todaro M, Merz $\mathrm{C}$, Rodermond $\mathrm{H}$, et al: Wnt activity defines colon cancer stem cells and is regulated by the microenvironment. Nat Cell Biol 12: 468-476, 2010.

18. Murata T, Mizushima H, Chinen I, Moribe H, Yagi S, Hoffman RM, Kimura T, Yoshino K, Ueda Y, Enomoto T, et al: HB-EGF and PDGF mediate reciprocal interactions of carcinoma cells with cancer-associated fibroblasts to support progression of uterine cervical cancers. Cancer Res 71: 6633-6642, 2011.

19. Zhu L, Cheng X, Ding Y, Shi J, Jin H, Wang H, Wu Y, Ye J, Lu Y, Wang TC, et al: Bone marrow-derived myofibroblasts promote colon tumorigenesis through the IL-6/JAK2/STAT3 pathway. Cancer Lett 343: 80-89, 2014.

20. Clapéron A, Mergey M, Aoudjehane L, Ho-Bouldoires TH, Wendum D, Prignon A, Merabtene F, Firrincieli D, DesboisMouthon C, Scatton O, et al: Hepatic myofibroblasts promote the progression of human cholangiocarcinoma through activation of epidermal growth factor receptor. Hepatology 58: 2001-2011, 2013.

21. Lewis MP, Lygoe KA, Nystrom ML, Anderson WP, Speight PM, Marshall JF and Thomas GJ: Tumour-derived TGF-betal modulates myofibroblast differentiation and promotes $\mathrm{HGF} /$ SF-dependent invasion of squamous carcinoma cells. Br J Cancer 90: 822-832, 2004.

22. Martin M, Wei $\mathrm{H}$ and $\mathrm{Lu} \mathrm{T}$ : Targeting microenvironment in cancer therapeutics. Oncotarget 7: 52575-52583, 2016.

23. Kawasaki H, Ohama T, Hori M and Sato K: Establishment of mouse intestinal myofibroblast cell lines. World J Gastroenterol 19: 2629-2637, 2013

24. Iwamoto T, Yamada K, Shimizu M and Totsuka M: Establishment of intestinal epithelial cell lines from adult mouse small and large intestinal crypts. Biosci Biotechnol Biochem 75: 925-929, 2011.

25. Yabe R, Miura A, Usui T, Mudrak I, Ogris E, Ohama T and Sato K: Protein phosphatase methyl-esterase PME-1 protects protein phosphatase $2 \mathrm{~A}$ from ubiquitin/proteasome degradation. PLoS One 10: e0145226, 2015.

26. Fujiwara N, Kawasaki H, Yabe R, Christensen DJ, Vitek MP, Mizuno T, Sato K and Ohama T: A potential therapeutic application of SET/I2PP2A inhibitor OP449 for canine T-cell lymphoma. J Vet Med Sci 75: 349-354, 2013

27. Usui T, Morita T, Okada M and Yamawaki H: Histone deacetylase 4 controls neointimal hyperplasia via stimulating proliferation and migration of vascular smooth muscle cells. Hypertension 63: 397-403, 2014

28. Livak KJ and Schmittgen TD: Analysis of relative gene expression data using real-time quantitative PCR and the 2(-Delta Delta C(T)) method. Methods 25: 402-408, 2001.

29. Le Rolle AF, Chiu TK, Zeng Z, Shia J, Weiser MR, Paty PB and Chiu VK: Oncogenic KRAS activates an embryonic stem cell-like program in human colon cancer initiation. Oncotarget 7: $2159-2174,2016$.

30. Hammond DE, Mageean CJ, Rusilowicz EV, Wickenden JA, Clague MJ and Prior IA: Differential reprogramming of isogenic colorectal cancer cells by distinct activating KRAS mutations. J Proteome Res 14: 1535-1546, 2015.
31. Vinante $\mathrm{F}$ and Rigo A: Heparin-binding epidermal growth factor-like growth factor/diphtheria toxin receptor in normal and neoplastic hematopoiesis. Toxins (Basel) 5: 1180-1201, 2013.

32. Rao GH, Liu HM, Li BW, Hao JJ, Yang YL, Wang MR, Wang XH, Wang J, Jin HJ, Du L, et al: Establishment of a human colorectal cancer cell line P6C with stem cell properties and resistance to chemotherapeutic drugs. Acta Pharmacol Sin 34: 793-804, 2013.

33. Rowehl RA, Burke S, Bialkowska AB, Pettet DW III, Rowehl L, Li E, Antoniou E, Zhang Y, Bergamaschi R, Shroyer KR, et al: Establishment of highly tumorigenic human colorectal cancer cell line (CR4) with properties of putative cancer stem cells. PLoS One 9: e99091, 2014.

34. Hung CF, Rohani MG, Lee SS, Chen P and Schnapp LM: Role of IGF-1 pathway in lung fibroblast activation. Respir Res 14: 102, 2013.

35. Theiss AL, Simmons JG, Jobin C and Lund PK: Tumor necrosis factor (TNF) alpha increases collagen accumulation and proliferation in intestinal myofibroblasts via TNF receptor 2 . J Biol Chem 280: 36099-36109, 2005.

36. Jobson TM, Billington CK and Hall IP: Regulation of proliferation of human colonic subepithelial myofibroblasts by mediators important in intestinal inflammation. J Clin Invest 101: 2650-2657, 1998

37. Yotsumoto F, Tokunaga E, Oki E, Maehara Y, Yamada $\mathrm{H}$, Nakajima K, Nam SO, Miyata K, Koyanagi M, Doi K, et al: Molecular hierarchy of heparin-binding EGF-like growth factorregulated angiogenesis in triple-negative breast cancer. Mol Cancer Res 11: 506-517, 2013.

38. Yahata Y, Shirakata Y, Tokumaru S, Yang L, Dai X, Tohyama M, Tsuda T, Sayama K, Iwai M, Horiuchi M, et al: A novel function of angiotensin II in skin wound healing. Induction of fibroblast and keratinocyte migration by angiotensin II via heparin-binding epidermal growth factor (EGF)-like growth factor-mediated EGF receptor transactivation. J Biol Chem 281: 13209-13216, 2006.

39. Kim JM, Bak EJ, Chang JY, Kim ST, Park WS, Yoo YJ and Cha JH: Effects of HB-EGF and epiregulin on wound healing of gingival cells in vitro. Oral Dis 17: 785-793, 2011.

40. Faria JA, de Andrade C, Goes AM, Rodrigues MA and Gomes DA: Effects of different ligands on epidermal growth factor receptor (EGFR) nuclear translocation. Biochem Biophys Res Commun 478: 39-45, 2016.

41. Kastanis GJ, Hernandez-Nazara Z, Nieto N, Rincón-Sanchez AR, Popratiloff A, Dominguez-Rosales JA, Lechuga CG and Rojkind M: The role of dystroglycan in PDGF-BB-dependent migration of activated hepatic stellate cells/myofibroblasts. Am J Physiol Gastrointest Liver Physiol 301: G464-G474, 2011.

42. Shi H, Lin B, Huang Y, Wu J, Zhang H, Lin C, Wang Z, Zhu J, Zhao Y, Fu X, et al: Basic fibroblast growth factor promotes melanocyte migration via activating PI3K/Akt-Rac1-FAK-JNK and ERK signaling pathways. IUBMB Life 68: 735-747, 2016.

43. Chun J and Kim YS: Platycodin D inhibits migration, invasion, and growth of MDA-MB-231 human breast cancer cells via suppression of EGFR-mediated Akt and MAPK pathways. Chem Biol Interact 205: 212-221, 2013.

44. Ong BA, Vega KJ and Houchen CW: Intestinal stem cells and the colorectal cancer microenvironment. World J Gastroenterol 20: $1898-1909,2014$ 